\title{
Las «Observaciones» del Real Colegio de Cirugía de la Armada en Cádiz (1742-1836)
}

\author{
Dr. Carlos Márquez Espinós \\ Académico de número \\ Real Academia de Medicina y Cirugía de Cádiz \\ ORCID: https://orcid.org/0000-0002-1830-1018
}

RESUMEN: Las «Observaciones» son un conjunto documental que acreditan las reuniones periódicas de los cirujanos del Real Colegio de Cirugía de Cádiz, en la segunda mitad del siglo XVIII y primeras décadas del siglo XIX, y que fueron el modelo a seguir por los Reales Colegios de Cirugía de Barcelona y Madrid.

Se conservan 310 documentos, el más antiguo data de 1742 y el último está fechado en 1836, que se presentaron en las Asambleas escolásticas del colegio gaditano constituyendo una novedad en la España de la Ilustración que, posteriormente, se incorporó en el colegio catalán y el madrileño. XIX.

Palabras clave: Observaciones, Real Colegio de Cirugía de Cádiz, España, Siglos XVIII-

\section{The «Observations» of the Royal College of Surgery of Cadiz (1742-1836)}

ABSTRACT: The «Observations» are a documentary set that accredits the periodic meetings of the surgeons of the Royal College of Surgery of Cádiz, in the second half of the 18th century and the first decades of the 19th century. It represented the model to be followed by the Royal College of Surgery of Barcelona and Madrid.

There are 310 documents preserved, the oldest one dates from 1742 and the latest from 1836. These documents were presented at the scholastic Assemblies of the Royal College of Surgery of Cadiz as a newness in the Spanish Enlightenment and afterwards were included in the Catalonian and Madrilenian Colleges.

Keywords: Observations, Royal College of Surgery of Cadiz, Spain, 18th-19th Century.

\section{INTRODUCCIÓN}

La existencia de una colección documental procedente del Archivo del Real Colegio de Cirugía de la Armada en Cádiz, que respondía al epígrafe «Observaciones», descubierta inicialmente por el Prof. Ferrer Fernández de la Riva en su Historia del Colegio ${ }^{1}$, que había

\footnotetext{
${ }^{1}$ Ferrer, D., 1961: Historia del Real Colegio de Cirugía de Cádiz. También, vid., una segunda edición, facsímil, con notas e índices de Márquez Espinós y Cabrera Afonso que fue impresa por el Servicio de Publicaciones de la Universidad de Cádiz, en 1983.

DOI del artículo:

https://doi.org/10.25267/Cuad investig fondos arch UCA.2021.i3.03 
que catalogar y estudiar, se convirtió en el primer trabajo de investigación histórico-médica que realicé dando lugar a mi Tesis de Licenciatura, dirigida por el Prof. Antonio Orozco Acuaviva, y que, a la postre, se convirtió en mi primera publicación de relevancia ${ }^{2}$.

Esta colección documental que hoy se conserva en los fondos del Archivo Histórico de la Universidad de Cádiz (AUCA) consta de 310 manuscritos datados entre los años 1742 a 1836. Los cuatro primeros son anteriores a la existencia del Real Colegio de Cirugía de Cádiz; dos fueron leídos en la Asamblea Amistoso-Literaria de Jorge Juan (1755 y 1756); otro procede del primer año de existencia del Colegio Nacional de Medicina y Cirugía (1837), heredero del Real Colegio de Cirugía de la Armada y, por último, un informe de cirugía forense emitido por la Junta de Maestros en 1815. Además, por los Libros de Actas del Colegio, que también se conservan en los fondos del Archivo Histórico de la UCA, conocemos que se leyeron otras 48 observaciones entre 1797 y 1835, cuyos manuscritos no se encuentran en esta serie por lo que no es descartable que en un futuro se pueda localizar alguna observación más.

Finalmente, una nota aclaratoria, la mayor parte de los manuscritos aparecen con la denominación de «Observaciones», aunque algunas veces se refieren a ellas como «Asambleas» y sólo en alguna ocasión como «Juntas Literarias» pero debido a la existencia de colecciones similares publicadas en Madrid por Lapuente Mateos ${ }^{3}$ el año 1949 como «Juntas Literarias» y por Usandizaga ${ }^{4}$, en 1956, que también se refirió a la colección existente en el Colegio de Barcelona como «Juntas Literarias» se adoptó esta denominación en la publicación a la que hemos hecho alusión anteriormente ${ }^{5}$.

\section{El Real Colegio de Cirugía de CÁdiz}

Los inicios y consolidación de la renovación científica en España tienen lugar en las últimas décadas del siglo XVII y primera mitad del siglo XVIIII. En primer lugar, desde un punto de vista institucional, surgieron la Regia Sociedad de Medicina y otras Ciencias de Sevilla (1700), la Academia de Ingenieros Militares de Barcelona (c.1715), la de Guardiamarinas de Cádiz (1717) y el Real Seminario de Nobles de Madrid (1726). Posteriormente, las novedades más importantes fueron los Reales Colegios de Cirugía de Cádiz (1748) y Barcelona (1760), la fundación del Observatorio de Marina de Cádiz (1753),

\footnotetext{
${ }^{2}$ Marquez Espinos, C., 1986: Las Juntas Literarias del Real Colegio de Cirugía de Cádiz. Catálogo de las «Observaciones» manuscritas (1742-1836).

${ }^{3}$ LAPUente Mateos, A., 1949 y 1951: «Juntas Literarias y censuras en el Real Colegio de San Carlos». También, sobre las asambleas del Real Colegio de San Carlos de Madrid, vid.: UsANDIZAGA, M., 1948: Historia del Real Colegio de Cirugía de San Carlos de Madrid (1787-1828), pp. 99-129; APARICIO SimÓn, J., 1956: Historia del Real Colegio de San Carlos de Madrid, pp. 168-220; BuRKE, M. E., 1977: The Royal College of San Carlos. Surgery and Spanish Medical Reform in the Late Eighteenth Century, pp. 124-129.

${ }^{4}$ Usandizaga, M., 1956: Juntas Literarias del Real Colegio de Cirugía de Barcelona, pp. 45-53. También, sobre las Juntas Literarias del Real Colegio de Cirugía de Barcelona, vid.: UsANDIZAGA SoRALUCE, M., 1964: Historia del Real Colegio de Cirugía de Barcelona (1760-1843). pp. 213-224; MASSSONS, J. M., 2002: Història del Reial Col-legi de Cirurgia de Barcelona, pp. 346-365; PÉREZ-PÉREZ, N., SiTGES-SERRA, A., 2010): «Juntas Literarias: legado de la cirugía ilustrada».

5 Cf.: Orozco Acuaviva, A. «Prólogo». En Márquez Espinós, C., 1986: Las Juntas Literarias del Real Colegio de Cirugía de Cádiz. Catálogo de las «Observaciones» manuscritas (1742-1836), pp. 9-10.
}

DOI del artículo: https://doi.org/10.25267/Cuad investig fondos arch UCA.2021.i3.03 
la Asamblea Amistoso-Literaria de Cádiz (1755), La Real Sociedad Militar de Madrid (1757), el Colegio de Artillería de Segovia (1762) o las Academias de Guardias de Corps de Madrid (1750), Artillería de Barcelona (1750) y de Ingenieros de Cádiz (1750).

En los años anteriores a la fundación del Real Colegio de Cirugía de la Armada (1748), la presencia de los cirujanos extranjeros, preferentemente franceses, llegados con Felipe V, se hizo notar en Cirugía. En este contexto, Jean Lacombe (castellanizado Juan Lacomba), nombrado Cirujano Mayor de la Armada en 1718 comenzó la renovación de la cirugía naval española. Deseaba incrementar los conocimientos de los cirujanos de los buques y para ello, construyó en 1728 un Anfiteatro Anatómico en el Hospital del Rey u Hospital de Marina de Cádiz; designó un catedrático demostrador anatómico (Gregorio de Condomina), formado en Montpellier, y obligó a asistir y practicar al hospital a todos los practicantes y cirujanos de los buques que llegaban a la ciudad. También, consiguió unas Ordenanzas para el Cuerpo de Cirujanos de Marina que normalizó el funcionamiento de éstos. Por último, se rodeó de cirujanos experimentados formados en Montpellier y París: Gaspar de Pellicer, Pedro Virgili; Francisco Nueve Iglesias y Francisco Canivell.

Sin embargo, para que esta renovación de los saberes sanitarios en la España del siglo XVIII se convirtiese en una realidad fue necesario que concurrieran dos circunstancias principales. En primer lugar, una «estrategia» ${ }^{6}$ que llevó a la implantación de los Reales Colegios de Cirugía; en Cádiz no existía Universidad y la Universidad de Sevilla quedaba distante, la hermandad de San Cosme y San Damián carecía de la fuerza suficiente para ser un obstáculo en el desarrollo de la institución ilustrada y la figura, así como las funciones, del Cirujano Mayor de la Armada obviaban el poder del Real Protomedicato. El Colegio de Barcelona, que se dedicaría a formar cirujanos para el ejército y estaría bajo la protección del Capitán General, tampoco tenía oposición por parte de la Universidad de Cervera. Finalmente, tras superar muchas dificultades, fue aprobado el Real Colegio de San Carlos, en 1787, para formar cirujanos que atendieran a la población civil, aunque durante los primeros años prácticamente no tuvo alumnos. En segundo lugar, en la creación del Real Colegio de Cirugía de la Armada, existió un «plan oculto» ${ }^{7}$ consistente en el aprendizaje, por los colegiales, de la medicina y la cirugía que se formaron no sólo para atender a la Armada o al Ejército sino también a la población civil, como se evidenció en el propio plan de estudios con el que se iniciaron sus enseñanzas y que quedó definido en la primera década de existencia del colegio gaditano ${ }^{8}$ y en donde se contempla, entre otras materias, la formación en partos; enfermedades de las mujeres y de los niños.

El 23 de noviembre de 1747, Pedro Virgili sustituyó a Juan Lacomba como Cirujano Mayor de la Armada y, en 1748, presentó un memorial para la creación en Cádiz de un colegio de cirujanos para la Marina que se hizo realidad gracias al apoyo del Marqués de la Ensenada. Se iniciaron los estudios en enero de 1749 en el propio hospital ya que el edificio del Real Colegio aún se estaba construyendo. Para las primeras plazas de colegiales, Virgili eligió a

\footnotetext{
${ }^{6}$ Orozco Acuaviva, A., 2001: «Los Reales Colegios de Cirugía en España».

${ }^{7}$ Ibídem.

${ }^{8}$ Orozco Acuaviva, A., 1988: «El modelo de enseñanza en el Real Colegio de Cirugía de Cádiz en el siglo XVIII».
}

DOI del artículo:

https://doi.org/10.25267/Cuad investig fondos arch UCA.2021.i3.03 
antiguos practicantes de cirugía con experiencia (más de tres años de antigüedad) y conocimientos de latín y filosofía para que con sólo tres años de formación pudieran salir a ejercer como cirujanos, aunque a partir de 1752 se obligó a los seis años de estudios que exigían los estatutos ${ }^{9}$.

En 1751, finalizó sus estudios la primera promoción; se enviaron tres alumnos a estudiar medicina a las Universidades de Leiden y Bolonia y ocho a la de París para perfeccionar sus estudios en partos; Oftalmología; Cirugía de la Piedra; Anatomía; Química; Física Experimental, etcétera $^{10}$. A su regreso a España algunos fueron nombrados profesores del Real Colegio.

La biblioteca ${ }^{11}$ se inició con la propia fundación del Real Colegio al considerar Pedro Virgili que ésta debía ser una parte fundamental del mismo. Para ello, inicialmente propuso como bibliotecario a Francisco Canivell, por su dominio del francés; del latín y su conocimiento del italiano; adquiriendo, ya desde 1749, libros de muy variada temática en Ámsterdam, Leiden, París y Londres. Los viajes y estancias de los alumnos pensionados en el extranjero serian aprovechados para realizar compras de libros que, posteriormente, remitirían a la institución. En este sentido, señaló Orozco ${ }^{12}$, la riqueza en libros médicos y quirúrgicos que existió en la biblioteca y la profusión en obras de materias poco acordes con el origen exclusivamente quirúrgico del Colegio pero que mostraron la oculta «vocación» médicoquirúrgica de sus fundadores. Además, hay que hacer notar la autorización de la Inquisición para que la Biblioteca «tuviera los libros prohibidos del Santo Oficio pertenecientes a Anatomía, Medicina, Botánica, Química, Física e Historia Natural, con la condición de que los libros estuvieran con la debida reserva y custodia» ${ }^{13}$.

Por Real Orden de 1757 se facultó al colegio para conceder a sus alumnos el grado de Bachiller en Filosofía (artes), privilegio que sólo poseían las universidades. Es a partir de esta fecha, señaló Orozco ${ }^{14}$, «cuando el colegio presenta su verdadera y novedosa imagen como centro superior a las propias universidades españolas y extranjeras, e incluso a sus modelos primitivos, las academias quirúrgicas de Montpellier y París, pues en Cádiz, se enseña, además de cirugía, medicina».

En los años siguientes se continuó el envío de colegiales al extranjero; se fundó en 1760 el Real Colegio de Cirugía de Barcelona y, en 1764, se publicaron las primeras ordenanzas comunes a los colegios de Cádiz y Barcelona.

A partir de 1768, Francisco Canivell, director del Colegio y Cirujano Mayor de la Armada, consiguió la concesión de uniforme para los colegiales y las distintas categorías de los

\footnotetext{
${ }^{9}$ Orozco Acuaviva, A., 2001: «Los Reales Colegios de Cirugía en España», pp. 387-388.

${ }^{10}$ Ibídem.

${ }^{11}$ Sobre este tema, Cf:: REMÓN RodRÍGUEZ, A., 2017: El libro médico-científico en la Biblioteca del Real Colegio de Cirugía de Cádiz. y GESTIDO DEL OlMo, R., 1994: Una Biblioteca ilustrada gaditana. Los fondos bibliográficos humanísticos del Real Colegio de Cirugía de la Armada.

12 Orozco Acuaviva, A., 1995: «La biblioteca de la Facultad de Medicina de Cádiz entre Europa y América», pp. 264-271.

${ }^{13}$ Ferrer, D., 1983. Historia del Real Colegio de Cirugía de Cádiz, pp. 115-116.

${ }^{14}$ Orozco Acuaviva, A., 2001: «Los Reales Colegios de Cirugía en España», pp. 385-392.
}

DOI del artículo:

https://doi.org/10.25267/Cuad investig fondos arch UCA.2021.i3.03 
cirujanos navales (1771); incrementó los laboratorios y la biblioteca; obligó al embarco de cirujanos en los navíos mercantes; estimuló la publicación de libros y redactó las ordenanzas de 1791, propias del colegio gaditano, en las que se hizo oficial la unión de la medicina y la cirugía.

El 23 de marzo de 1805, Carlos Francisco Ameller fue nombrado Cirujano Mayor de la Armada y, por consiguiente, se convirtió en director del Colegio. Le tocó vivir circunstancias difíciles como el hundimiento de la Marina española en Trafalgar ${ }^{15}$, en octubre de ese año; la Guerra de la Independencia con los franceses ${ }^{16}$; el periodo constituyente de las Cortes que dio lugar al nacimiento de la primera constitución española; el comienzo de la independencia de las colonias americanas ${ }^{17} \mathrm{y}$, por último, la fundación de la Sociedad Médico-Quirúrgica de Cádiz estrechamente ligada a la vida cotidiana del colegio ${ }^{18}$.

El Colegio dejó de pertenecer a la Marina en 1833 y es su último director Francisco de Flores Moreno ${ }^{19}$ hasta el año 1836. El curso siguiente nació para la enseñanza en Cádiz el Colegio Nacional de Medicina y Cirugía, sucesor del Real Colegio de Medicina y Cirugía de la Armada ${ }^{20}$.

\section{Plan de Estudios}

En general, las materias que se cursaron se podían clasificar de la siguiente forma, siguiendo las Ordenanzas de 1791 propias del Colegio de Cádiz y vigentes a partir de $1805^{21}$ :

En primer lugar, estudios básicos, que englobaron, en el primer año, Anatomía; Física Experimental; Química; Botánica y Vendaje; mientras que el segundo año constaría de Fisiología; Higiene; Patología General; Terapéutica y Materia Medicinal.

En segundo lugar, estudios propios de cirugía, que corresponderían al exclusivo ejercicio profesional del cirujano. En estos estuvieron comprendidas materias que se impartían en el tercer año como la patología propia de la Cirugía, distinguida en tratados de tumores, úlceras, heridas de toda especie, enfermedades de los ojos y algunas particularidades de los dientes y el Álgebra Quirúrgica. En el cuarto año, el modo de practicar las operaciones de cirugía, haciendo que se ejercitaran en ellas los alumnos sobre los cadáveres; los tratados de partos, de

\footnotetext{
${ }^{15}$ Sobre la actuación del Real Colegio en Trafalgar, Vid.: CABRERA AFONSO, J. R., 2006: «El Real Colegio de Cirugía de Cádiz y el combate de Trafalgar», pp. 253-275 y ARAGÓN ESPESO, M., 2006: Cádiz, la medicina y la cirugía en la época de Trafalgar.

${ }^{16}$ Cf.: PÉrez Pérez, A., MÁrquez Espinos, C., 2014: La medicina en la época de las Cortes de Cádiz, pp. $425-$ 440.

${ }^{17}$ PÉrez PÉRez, A., MÁrquez EsPinos, C., 2009: Juan Miquel y Salazar (1792-1866). Portorrealeño y pionero de la medicina chilena.

18 Vid.: Márquez Espinos, C., 2009: La formación médica de Juan Miquel y Salazar (1792-1866) en el Cádiz de las Cortes, pp. 61-63 y MÁRQuez EsPINós, C., 1986: Las Juntas Literarias del Real Colegio de Cirugía de Cádiz. Catálogo de las «Observaciones» manuscritas (1742-1836), pp. 31-46.

${ }^{19}$ Para su biografía, vid.: VACA HeRnándeZ, M., 2015: Vida y obra de Don Francisco de Flores Moreno.

${ }^{20}$ Ferrer, D., 1983: Historia del Real Colegio de Cirugía de Cádiz, p. 261.

${ }^{21}$ ORDENANZAS, 1791, p. 45.

DOI del artículo:

https://doi.org/10.25267/Cuad investig fondos arch UCA.2021.i3.03 
mujeres paridas y niños recién nacidos y las enfermedades venéreas. También, se añadió el estudio de la Cirugía Legal.

En tercer lugar, estudios de doctrina médica que se impartieron en el quinto curso y se repetían el sexto: el tratado de afectos de medicina, la exposición de los aforismos de Hipócrates y una especial explicación de las enfermedades castrenses y concretamente de las de los navegantes.

El estudio de estas materias se introducía progresivamente, con ligeras matizaciones según el plan de estudios vigente en la época.

Orozco $^{22}$ indicó que de estos conocimientos son rigurosas novedades en la enseñanza en España, Física Experimental, Química, Materia Médica y Enfermedades de los Navegantes. Añadió que constituyen novedad la enseñanza con personalidad propia, separada de los textos generales en que habitualmente se incluían; Heridas por Armas de Fuego, Vendajes, Enfermedades de los Ojos, Enfermedades Venéreas, Enfermedades de las Mujeres y Niños e Higiene.

Se justificó desde un principio la existencia de estas materias en el plan de estudios en la necesidad de que los cirujanos realizaban su actividad profesional en buques de guerra que solían llevar pasaje, viajaban mujeres y niños, y, también, ejercían su labor en los buques mercantes de la Carrera de Indias que igualmente llevaban pasaje. Además, estos cirujanos atendían a la población civil o militar de los puertos y apostaderos, por lo que el conocimiento de estas materias quedaba más que justificado.

Este plan de estudios se consolidó con las Ordenanzas de 1791 que consagraron la unión de la Medicina y la Cirugía y

... si el Director y la Junta de Maestros no advirtiesen en algunos alumnos al fin del cuarto año, toda la instrucción debida en las materias de Cirugía, los detendrán otro más, para perfeccionarse en aquellas de que tengan más necesidad y adquirir la soltura correspondiente, sin pasarlos al estudio de las enfermedades de Medicina que se enseñarán, como queda dicho, en el quinto y sexto año ... ${ }^{23}$

Además, con estas Ordenanzas, el Real Colegio de Medicina y Cirugía de Cádiz fue la primera institución no universitaria europea que otorgó Grados de Bachiller y Licenciado en Medicina y Cirugía.

\section{RÉGIMEN DE ENSEÑANZA}

Una vez admitido el colegial; el curso comenzaba el mes de octubre y finalizaba en julio. El mes de agosto era de vacaciones y en septiembre se celebraban los exámenes generales correspondientes al año en curso. Los exámenes de septiembre eran calificados de generales

\footnotetext{
22 OrozCo Acuaviva, A., 1988: «El modelo de enseñanza en el Real Colegio de Cirugía de Cádiz en el siglo XVIII».

${ }^{23}$ ORDENANZAS, 1791, p. 45.

DOI del artículo:

https://doi.org/10.25267/Cuad investig fondos arch UCA.2021.i3.03 
por el hecho de que mientras en el primer curso sólo había que rendir sobre las cuatro primeras asignaturas del plan de estudios, en el sexto curso, la evaluación en Junta de Catedráticos correspondía al conjunto de todas las materias del plan de estudios independientemente del año en que se había cursado, aunque esto cambiaria con el tiempo.

En el Colegio la disciplina fue militar y, de forma general, la distribución del tiempo a lo largo del día fue la siguiente:

\section{Por la mañana:}

- Estudio

4 a $6 \mathrm{~h}$

- Curas y hacer los tópicos

6 a $9 \mathrm{~h}$

- Disecar, los de anatomía

9 a $10,30 \mathrm{~h}$

- Clases

9 a $12 \mathrm{~h}$

- Cortar lienzo en festivos y vacaciones $10 \mathrm{~h}$

- Comida

12 a $13,30 \mathrm{~h}$

2. Por la tarde y noche:

- Cortar lienzo en días de clases

13,30 a 14 h.

- Disecar, los de anatomía

14,30 a Oración

- Clases hasta mayo

14,30 a $16 \mathrm{~h}$

- Clases, lo demás

18 a $19 \mathrm{~h}$

- Estudio, hasta mayo

18 a $20 \mathrm{~h}$

- Estudio, lo demás

16 a $18 \mathrm{~h}$

- Cena hasta mayo

$20 \mathrm{~h}$

- Cena, lo demás

$20,30 \mathrm{~h}$

- Silencio, hasta mayo

$22 \mathrm{~h}$

— Silencio, lo demás

$22,30 \mathrm{~h}$

Finalmente, como resumió Orozco ${ }^{24}$, «diariamente, excepto los jueves y domingos, tras la correspondiente clase, los alumnos tenían que sostener la lección del día anterior. Los jueves exponía en lengua latina uno de los colegiales y era censurado por compañeros. Por la tarde se presentaban las observaciones, clínicas o doctrinales, como se hacía en las sesiones de la Real Academia de Cirugía de París».

\section{LAS «OBSERVACIONES»: DESARROLLO NORMATIVO}

Ya en el memorial que entregó Virgili al Marqués de la Ensenada, el 29 de mayo de 1748, proponiendo la fundación de un Colegio, «... en el cual se enseñe la Cirugía con el método

${ }^{24}$ Orozco Acuaviva, A., 2001: «Los Reales Colegios de Cirugía en España», pp. 387-388.

DOI del artículo:

https://doi.org/10.25267/Cuad investig fondos arch UCA.2021.i3.03 
que se requiere, deduciendo sus doctrinas de los experimentos físicos, observaciones y experiencia práctica $\ldots\rangle^{25}$, establecía con respecto a las Observaciones:

... siempre que se hallen en Cádiz los Ayudantes deberán asistir al Hospital [...] procurando formalizar todas las Observaciones de los casos que fuesen dignos de ellos y conspiren a la propia enseñanza a cuyo fin guardando su turno, según dispusiere el Cirujano Mayor se ha de leer una todas las semanas en el día que a este efecto determinare, y en el cual deberán concurrir todos los Ayudantes a la hora que infaliblemente citare dicho Cirujano Mayor y habiéndose leído y examinado en todas sus partes por dicho Director y sus Ayudantes, se escribirá con toda difusión en un Libro que se debe determinar para ello a fin de que en todo tiempo conste y sea de grande adelantamiento y utilidad ... ${ }^{26}$.

Posteriormente, esta declaración de intenciones fue confirmada en la orden de noviembre de ese año por la que se estableció el Real Colegio de Cirugía de la Armada en Cádiz que además recogió la necesidad de que existiera un salón para las conferencias y lecciones de las «Observaciones» de Cirugía ${ }^{27}$. Aun así, conocemos por una carta de Francisco Nueve Iglesias a Máximo Dubouchet (Ministro Inspector del Real Hospital) que inicialmente el desarrollo de las asambleas con las lecturas de las observaciones se llevó a cabo en el Anfiteatro Anatómico ya que el edificio del Colegio no estaba terminado ${ }^{28}$.

Por esta época, vieron la luz las Adiciones a las Ordenanzas de Cirujanos de Marina de 1728, transcritas por Clavijo ${ }^{29}$, que obligaban a los cirujanos embarcados a llevar en un cuaderno anotadas las «Observaciones» que hiciesen durante la navegación. De esta forma, se estableció un tipo de observación que solo se dio en el Colegio de Cádiz: las enviadas por los cirujanos embarcados.

El día 13 de julio de 1751, Pedro Virgili propuso que se hiciera imprimir el método con que se debía proceder para hacer las observaciones, en vista de que se habían presentado algunas $« \ldots$ sin que las acompañara cosa con orden...»" ${ }^{30}$ y el 20 de agosto entregó a los colegiales destinados a París unas instrucciones en las que les exhortó a asistir a la curación

\footnotetext{
25 «Año de 1748. Representazion hecha por $D^{n}$ Pedro Virgili para establecer el Real Colegio». Cf:: Archivo de la Universidad de Cádiz (AUCA). Libro [Copiador] de Reales órdenes y decretos[...] en favor del Real Hospital Cirugía, Real Colegio Seminario de Cádiz e individuos que lo componen, L-54 RC, p. 74.

${ }^{26}$ Ibídem, pp. 76-77.

${ }^{27}$ «Orden dada en este año para el establecimiento del Real Colegio de Cirujia». Cf:: Archivo de la Universidad de Cádiz (AUCA), Libro [Copiador] de Reales órdenes y decretos[...] en favor del Real Hospital Cirugía, Real Colegio Seminario de Cádiz e individuos que lo componen,-L-54 RC, pp. 85-86.

28 «Respuesta a una orden de 9 de agosto de 1749» fechada en «Cádiz y agosto 11 de 1749». Cf.: MÁRQUEZ EsPINós, C., 1986: Las Juntas Literarias del Real Colegio de Cirugía de Cádiz. Catálogo de las «Observaciones» manuscritas (1742-1836), p. 205.

${ }^{29}$ Clavijo y Clavijo, S., 1925: Historia del Cuerpo de Sanidad Militar de la Armada. p. 160.

${ }^{30}$ Cf:: Archivo de la Universidad de Cádiz (AUCA), Libro de Noticias de propuestas y deliberaciones, así como de los gastos del Real Colegio de Cirugía, L-22-RC, p. 2.

DOI del artículo:

https://doi.org/10.25267/Cuad investig fondos arch UCA.2021.i3.03 
de las enfermedades «con mucha puntualidad y cuidado» y enviaran las observaciones de ellas «con claridad y difusión» ${ }^{31}$.

Al año siguiente, el 1 de abril, el impresor Nicolás de Quintana entregó a D. Francisco Canivell, Bibliotecario del Colegio, 500 pliegos con el título Methodo que deben observar los Cirujanos de la Real Armada y Colegiales del Real Collegio de Cirujia para formar las observaciones de los casos particulares que se distribuyeron gratis entre los cirujanos y colegiales $^{32}$. Lamentablemente, hasta el momento presente no tenemos noticias de que se haya localizado algún ejemplar de este impreso que probablemente disiparía algunas incógnitas que tenemos sobre la realización de las «Observaciones».

Años más tarde, el 13 de octubre de 1757, Virgili estableció normas precisas para la realización de las censuras, que se llevarían a cabo por «dos comisarios» que darían noticia de la observación el jueves de la siguiente Asamblea y, además, estableció que cualquiera podría criticarla «aún después del día de su última lectura; siéndole igualmente libre ejecutarlo de palabra o por escrito» ${ }^{33}$.

El 13 de abril de 1758, Pedro Virgili fue nombrado Cirujano de Cámara y se trasladó a Madrid; el cargo de Cirujano Mayor de la Armada quedó vacante, aunque Virgili retuvo por Real Orden, de 2 de mayo de 1758, la Dirección del Colegio. Pedro Virgili ya no volvería a Cádiz, aunque siguió siendo director del Real Colegio e incluso simultaneó esta Dirección con la fundación y dirección del Real Colegio de Cirugía del Ejército en Barcelona, el año 1760, en un caso histórico bien documentado de pluriempleo, por parte de un profesional de las ciencias médicas, en la Administración española. Probablemente, en esta circunstancia esté la clave por la que las Ordenanzas de 1764 fueron comunes a ambos colegios. A Francisco Nueve Iglesias se le nombró, el 4 de mayo de 1758, Cirujano Mayor de la Armada y se le hizo el encargo complementario de mantener la actividad cotidiana del colegio de acuerdo a la normativa existente.

En consecuencia, Francisco Nueve Iglesias continuó la labor de Pedro Virgili en el colegio gaditano, pero de una forma sui generis pues Pedro Virgili siguió siendo el director, aun cuando ejerció su labor a distancia. Esta anómala situación no debió beneficiar la labor docente realizada en el Colegio como se desprende de la carta que recibió el Secretario del colegio, a la sazón Francisco López Cárdenas, el 18 de septiembre del año 1759, remitida por Pedro Virgili, con instrucciones precisas para la realización de las clases y las asambleas. En esta misiva, Virgili especificó que las asambleas se realizarían todos los jueves, exceptuando las semanas que llevaren fiestas de precepto; en invierno a las dos y en verano a las tres de la

\footnotetext{
${ }^{31}$ «Copia de la instrucc. ${ }^{n}$ dada a los Coleg. ${ }^{s}$ que están destinados a Paris por el Ciruj. ${ }^{o}$ Maior de la Arm. ${ }^{d a}$ D. ${ }^{n}$

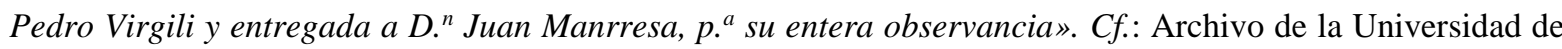
Cádiz (AUCA), Libro [Copiador] de Reales órdenes y decretos[...] en favor del Real Hospital Cirugía, Real Colegio Seminario de Cádiz e individuos que lo componen, L-54 RC, p. 205.

${ }^{32}$ Cf: : Archivo de la Universidad de Cádiz (AUCA). Libro de Noticias de propuestas y deliberaciones, así como de los gastos del Real Colegio de Cirugía, L- 22 RC, pp. 4-5.

33 «Methodo Instructivo q. ${ }^{e}$ según la mente de nuestro Director deben observar en sus Clases y Conferencias los Maestros de este R. ${ }^{l}$ Collegio». Cf.: MÁRQuez Espinós, C., 1986: Las Juntas Literarias del Real Colegio de Cirugía de Cádiz. Catálogo de las «Observaciones» manuscritas (1742-1836), pp. 206-208.
}

DOI del artículo: https://doi.org/10.25267/Cuad investig fondos arch UCA.2021.i3.03 
tarde; tendrían una duración de dos horas y los días que no haya observación, «se leerán unas memorias sobre puntos prácticos de Cirugía que deberán trabajar por su turno los Maestros», encargándole al Secretario, Francisco López Cárdenas, que hiciese una lista puntual de los que faltasen, «... la que se me remitirá cada semana» ${ }^{34}$ De esta forma, además de fijar normas muy precisas para el desarrollo de las Asambleas quedó establecido el tercer tipo de observación, que también se producirá en los Reales Colegios de Cirugía de Barcelona y Madrid; nos referimos a la observación sobre un punto concreto de doctrina sin necesidad de historia clínica.

Volvió a insistir Virgili, en octubre de 1763, a Francisco López Cárdenas con otra carta de seis puntos ${ }^{35}$ que refundió todas la normativa que había estado dictando sobre la manera de realizar las asambleas y los distintos tipos de observaciones; así, el punto primero estableció definitivamente los jueves como día de observación; que todos los Maestros comenzando por el Cirujano Mayor presentaran por turno una observación «adornada de reflexiones sobre todos sus puntos» o una «disertación teórico-práctica sobre la materia que le tocase por suerte» y acabada la lectura todos los Maestros darían su dictamen empezando por el más moderno. En el punto segundo, instituyó que «si el caso propuesto fuese arduo», se nombrarían dos Maestros para que presentaran su censura en la asamblea siguiente. En el tercero, recordó la obligación que tenían de asistir a las asambleas todos los Colegiales y, también, los Cirujanos Primeros y Segundos desembarcados. En el cuarto punto, les exhortó para que velaran por la obligación que tenían los cirujanos embarcados de recopilar y presentar en Junta las observaciones «de los casos curiosos que ocurren durante la navegación» y, por último, en el sexto estableció que se diera publicidad, a las materias que tenía que tratar cada uno, en un tablón de anuncios situado en la Biblioteca.

Aunque Francisco Nueve Iglesias, por medio de López Cárdenas le respondió «... se continuará con exactitud la observancia...» ${ }^{36}$, Pedro Virgili no se dio por satisfecho y volvió a insistir a Francisco López Cárdenas «Vd. me avisará de las omisiones que observase, como así mismo todos los meses me dará parte de las memorias y observaciones, que se hubieren leído en las asambleas...» ${ }^{37}$.

Este modelo de instrucción para los alumnos fue exportado por Pedro Virgili, procedente de Cádiz, a Barcelona y, más tarde, se impuso en el Real Colegio de San Carlos de Madrid por lo que, a partir de 1764, todas las normas para la celebración de las asambleas; juntas literarias o lectura de observaciones, serían recogidas por las distintas Ordenanzas de los Reales Colegios de Cádiz, Barcelona y Madrid.

\footnotetext{
34 «Carta de D. ${ }^{n}$ Pedro Virgili a D. ${ }^{n}$ Fran. ${ }^{c o}$ Lopez». Cf.: MÁRQuez EsPinós, C., 1986: Las Juntas Literarias del Real Colegio de Cirugía de Cádiz. Catálogo de las «Observaciones» manuscritas (1742-1836), pp. 208-209.

35 «Carta de D. ${ }^{n}$ Pedro Virgili a D. ${ }^{n}$ Fran. ${ }^{c o}$ Lopez Cardenas. Secret. ${ }^{\circ}{ }$. Cf.: MÁRQuez Espinós, C., 1986: Las Juntas Literarias del Real Colegio de Cirugía de Cádiz. Catálogo de las «Observaciones» manuscritas (17421836), pp. 210-212.

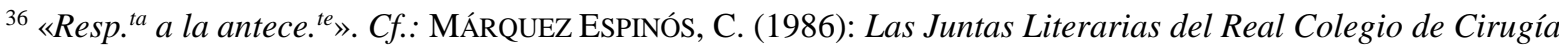
de Cádiz. Catálogo de las «Observaciones» manuscritas (1742-1836), pp. 212-213.

${ }^{37}$ «Carta de D. ${ }^{n}$ Pedro Virgili al Secret. ${ }^{o}$ del Colleg. ${ }^{\circ} »$. Cf.: MÁRQueZ EsPinÓs, C. (1986): Las Juntas Literarias del Real Colegio de Cirugía de Cádiz. Catálogo de las «Observaciones» manuscritas (1742-1836), p. 213.
} 
En 1764, se publicaron las Ordenanzas comunes a los Reales Colegios de Cirugía de Cádiz y Barcelona que sólo aportaron como novedad la obligación de realizar las censuras a todas las observaciones y no sólo a las «más arduas» ${ }^{38}$. También, este mismo año, en el Colegio hubo una permuta de secretario, hecho del que quedó constancia en los Libros de Actas y por el que sabemos que existieron dos Libros de Observaciones ${ }^{39}$, que en la actualidad están desaparecidos.

Este mismo año, en el mes de septiembre, se reunió el Claustro del Colegio de forma extraordinaria bajo la presidencia de José de Nájera, sustituto de Francisco Nueve Iglesias por fallecimiento de éste, reflejando el Acta lo tratado sobre el desarrollo de las Asambleas escolásticas y la lectura de las observaciones:

Todos los jueves a las 3 de la tarde en invierno, y a las 4 en verano, presentará cada Maestro por su antiguiedad una observación teórico-práctica, en la que se controvertirán los puntos de doctrina, que ocurrieren, y se consideraren más útiles para la instrucción de la juventud, y progreso de la ciencia.

Estas observaciones serán censuradas con toda moderación por Comisarios, que para ello nombrará el Vicepresidente, o su Teniente, para el jueves próximo, según está mandado por nuestro Director en 13 de octubre de 1751 con convenio de todos los Maestros y como más largamente consta en su carta orden datada en San Ildefonso a 5 de octubre de 1763. Estas memorias, sus censuras, y las oraciones inaugurales se entregarán al Secretario para que las archive, y haga anotar en el Libro correspondiente, y se previene, que a fin que no llegue a verificarse el que falte asunto de que tratar por ausencia o enfermedad del Maestro a quien perteneciere, deberá el que por su antigüedad se siguiere, tener pronta, y llevar consigo la pieza, que deberá presentar a la semana siguiente, con una semana de anticipación en el día mismo de Asamblea, pero si otras que los Maestros presentaren alguna observación, se le dará lugar, suspendiendo el maestro a quien tocare la lectura de la suya para más adelante ${ }^{40}$.

Toda esta normativa interna será recogida de forma oficial con la publicación, en 1791, de las nuevas Ordenanzas por las que el colegio se denominó desde esta época Real Colegio de Medicina y Cirugía ${ }^{41}$. Por otra parte, la Armada en sus nuevas Ordenanzas Generales, en los artículos 11 y 24, les recordó a los médicos-cirujanos embarcados la obligación que tenían de enviar observaciones ${ }^{42}$.

\footnotetext{
${ }^{38}$ Estatutos, 1764, pp. 9-10.

39 «Mutación de Secretario». Cf.: MÁrquez EsPINÓs, C., 1986: Las Juntas Literarias del Real Colegio de Cirugía de Cádiz. Catálogo de las «Observaciones» manuscritas (1742-1836), pp. 213-214.

40 «Actas, ô acuerdos de la Asamblea extraordinaria celebrada en el día 24 de Septiembre de 1764». Cf.: MÁrquez Espinós, C., 1986: Las Juntas Literarias del Real Colegio de Cirugía de Cádiz. Catálogo de las «Observaciones» manuscritas (1742-1836), pp. 214-217.

${ }^{41}$ ORDENANZAS, 1791, pp. 35-51.

${ }^{42}$ Clavijo y Clavijo, S., 1925: Historia del Cuerpo de Sanidad Militar de la Armada, pp. 210-211.
}

DOI del artículo: 
Posteriormente, la Junta Superior Gubernativa, recién creada y residente en la Corte, obligó al colegio de Cádiz a regirse por las Ordenanzas del Colegio de Barcelona de 1795. La inmediata protesta, ante esta injusticia, por parte del Director (José Selvareza) y del Cirujano Mayor de la Armada (Vicente Lubet) provocó la destitución de ambos ${ }^{43}$ y el nombramiento de Domingo Vidal, procedente del Real Colegio de Barcelona, como nuevo Director y Cirujano Mayor de la Armada que tomó posesión en agosto de 1796.

En lo que respecta a la celebración de las asambleas, estas Ordenanzas de 1795 aportaron varias normas, de entre las que destacamos las siguientes:

leídas las censuras [...] deberán todos los miembros de la Juntas, sin excusa, exponer públicamente su dictamen por orden de antigüedad, empezando por el más moderno [...]. Después de la sesión y conferencia [...] resumirán los Catedráticos sus dictámenes, para que el Secretario los anote al pie de la censura, ...

Recogieron la necesidad del censor para que revisando la observación «forme un pequeño extracto en que nada se omita de lo sustancial» y, por último, establecieron que «una de las principales obligaciones de la Junta ha de ser la publicación de estas obras, que procurará se verifique con la posible brevedad ...» ${ }^{44}$.

Las últimas normas, en lo que se refiere a las Asambleas, ya aparecieron en el siglo XIX; en 1821, el Reglamento para los Agregados a la Escuela ${ }^{45}$, que en su artículo séptimo equiparó a éstos con los Catedráticos en la forma y obligación de presentar observaciones en las asambleas escolásticas; en 1827, el Reglamento para el Gobierno de los Colegios de Medicina y Cirugía que vio la luz en $1828^{46}$ no aportó novedades y, en 1832, un oficio de la Real Junta Superior Gubernativa, del 25 de octubre,

... previniendo que las Juntas literarias que deben celebrarse todos los jueves en los Reales Colegios de la Facultad según el párrafo $3^{\circ}$, Capítulo $5^{\circ}$ del Reglamento que lo rige sean públicas como en él se previene, siempre que no se verifique así en la actualidad...

A lo que la Junta del colegio gaditano contestó «... que siempre dichas Juntas han sido públicas en este Real Colegio» ${ }^{47}$.

\footnotetext{
${ }^{43}$ FERRER, D., 1966: «De la unión del estudio de la Medicina y la Cirugía».

${ }^{44}$ ORDENANZAS, 1795, pp. 10-16.

45 «Reglamento formado.$^{r}{ }^{r}$ acuerdo de la Junta, . $^{a}$ los agregados a la Escuela». Cf.: Archivo Histórico de la Universidad de Cádiz (AUCA), Libro [tercero] de actas de la [Junta] del Real Colegio de Cirugía y Real Colegio de Medicina y Cirugía, años 1825-1832, L-24-RC, pp. 18-21.

${ }^{46}$ REAL CÉDUlA, 1828, pp. 21-26.

${ }^{47}$ «Sesión Extraordinaria del 2 de noviembre de 1832». Archivo Histórico de la Universidad de Cádiz (AUCA), Libro [cuarto] de [Actas] de la Junta del Colegio Nacional de Medicina y Cirugía, años: 1832-1836. [libro Cuarto], L-25 RC, pp. 13-14.

DOI del artículo: https://doi.org/10.25267/Cuad investig fondos arch UCA.2021.i3.03 


\section{LAS «OBSERVACIONES»: ORIGEN, TIPOS Y FINALIDADES}

Las «Observaciones» fueron un instrumento fundamental en la enseñanza médica del siglo XVIII europeo por lo que fue utilizada en Cádiz para el aprendizaje de los cirujanos en una fecha anterior a la fundación del Real Colegio de Cirugía por Juan Lacomba.

Una vez instituido el Colegio, este tipo de enseñanza fue parte fundamental de las Asambleas escolásticas o Juntas Literarias de los jueves por la tarde y en las que podemos distinguir, atendiendo sus características intrínsecas y a su contenido, los siguientes tipos:

1. Observaciones con historia clínica (figs.1-5), que siguen el modelo de la Observatio clásica.

Esta Observatio clásica es el resultado de la evolución de los consilium medievales ya fuera el consilium pro, redactados para la formación terapéutica, o el consilium de, redactados para la formación clínica del lector. La evolución y depuración de los consilium dará lugar a la Observatio renacentista que además de enseñar a «saber hacer», como el consilium, pretende enseñar a «saber ver» y a «saber entender» la enfermedad en el individuo. En los siglos XVI y XVII, la práctica de autopsias para comprobar de qué había fallecido el paciente dio lugar a que se incorporaran a la Observatio renacentista protocolos de autopsia, convirtiéndose ésta en Observatio anatomoclínica. Finalmente, será Hermann Boerhaave (1668-1738) el que dará lugar a la constitución definitiva del «canon de la historia clínica», al publicar las historias clínicas del barón Juan de Wassenaer (1724) y del marqués de SaintAuban (1728), y que reducida a sus partes esenciales se haya integrada por $^{48}$ :

I. Descriptio subjecti: nombre, edad, condición social, idiosincrasia del sujeto.

II. Praegressa: antecedentes remotos y próximos del paciente.

III. Status praesens: estado del enfermo cuando recibe la primera visita del médico.

IV. Cursus morbi: curso de la enfermedad después de la primera visita del médico y de instituido el tratamiento.

V. Inspectio cadaveris: examen anatomopatológico del cadáver si el fin de la enfermedad ha dado lugar a la muerte del enfermo.

${ }^{48}$ LaÍn EnTRALGO, P., 1961: La Historia clínica. Historia y teoría del relato patográfico, p.102.

DOI del artículo:

https://doi.org/10.25267/Cuad investig fondos arch UCA.2021.i3.03 
Figura 1: Observación $n^{\circ}{ }^{\circ}$ 20. «Observacion de una Castracion, ô extirpacion del teste â causa de una supuracion putrida en el; haviendo precedido un antiguo sarcocelle; por el Cirujano $\mathrm{M}^{\text {or }}$ de la Armada $\mathrm{D}^{\mathrm{n}}$ Pedro Virgili». [1754].

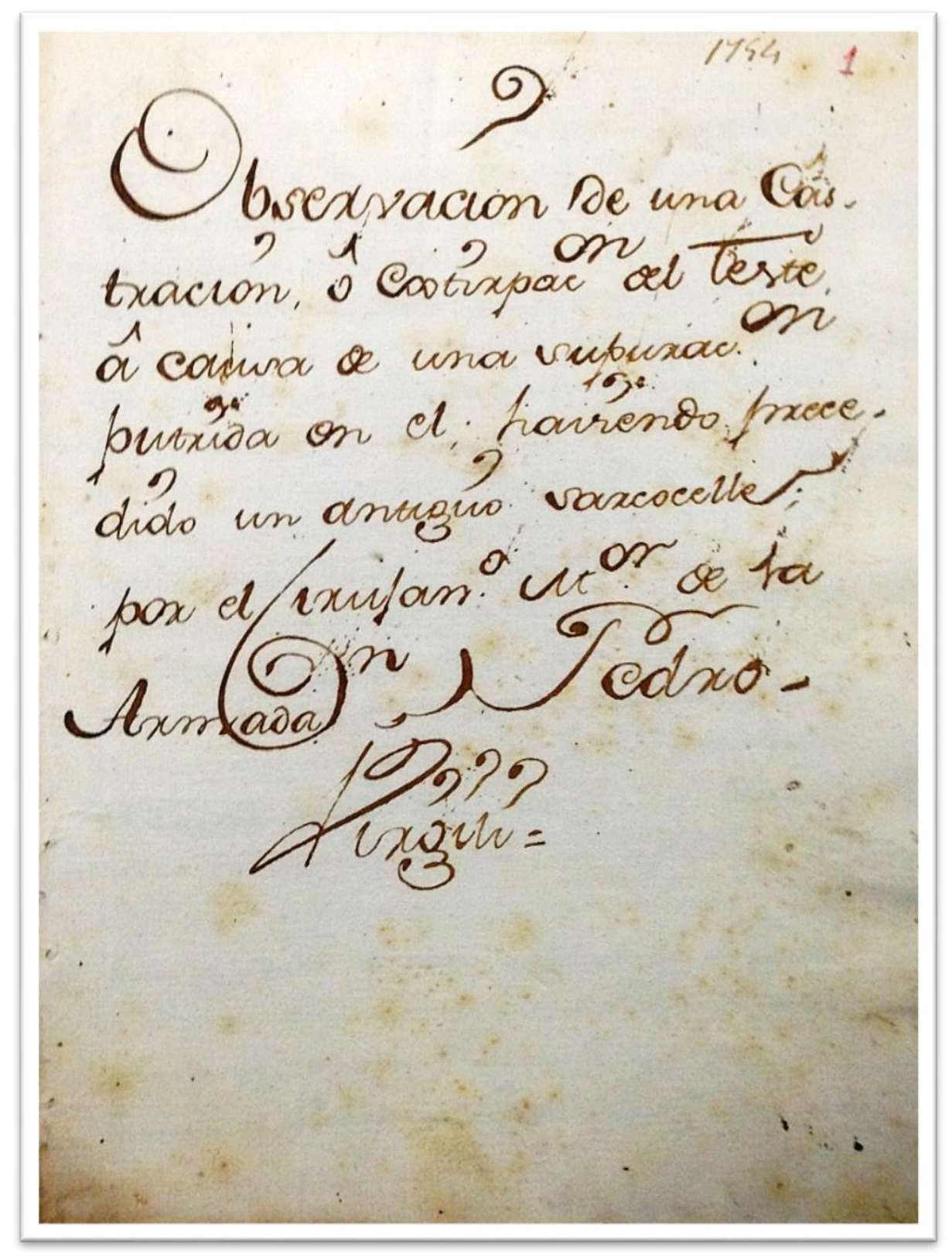

Fuente: Archivo de la Universidad de Cádiz (AUCA), C-35-20 RC 
Figura 2: Observación $n .^{o}$ 42. «Observ ${ }^{\text {on }}$ sobre una herida de cabeza felizm ${ }^{\text {te }}$ curada por medio de la trepanacion, comunicada $\mathrm{p}^{\mathrm{r}} \mathrm{D}^{\mathrm{n}}$ Fran $^{\mathrm{co}}$ Villaverde». «Cadiz y enero 18 de 1766»

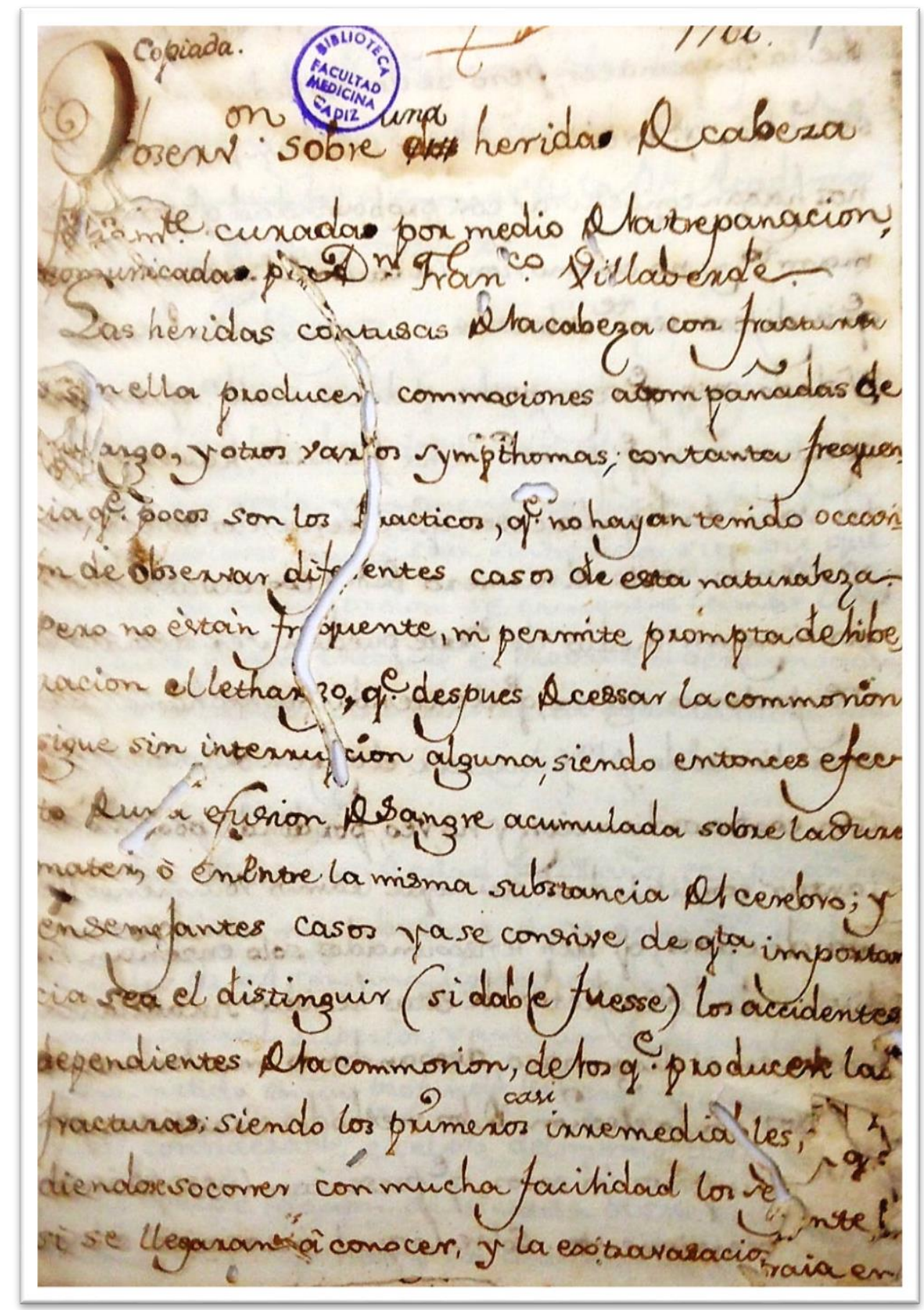

Fuente: Archivo de la Universidad de Cádiz (AUCA), C-35-42 RC

DOI del artículo:

https://doi.org/10.25267/Cuad investig fondos arch UCA.2021.i3.03 
Figura 3: Observación $n .^{\circ}$ 99. «Reflecciones â cerca del Methodo Curativo de la herida que padecio el $\mathrm{S}^{\mathrm{r}}$. $\mathrm{D}^{\mathrm{n}}$. Antonio Barcelot comandante de los Javeques, y dirigida al $\mathrm{S}^{\mathrm{r}}$. $\mathrm{D}^{\mathrm{n}}$. Francisco Canivell Cirujano Maior de la $\mathrm{R}^{1}$. Armada y Vice-Presidente del $\mathrm{R}^{1}$. Collegio de Cirugia en Cadiz». Por Francisco Ignacio Bousquett en «Menorca y Diciembre a 23 de $1770 »$.

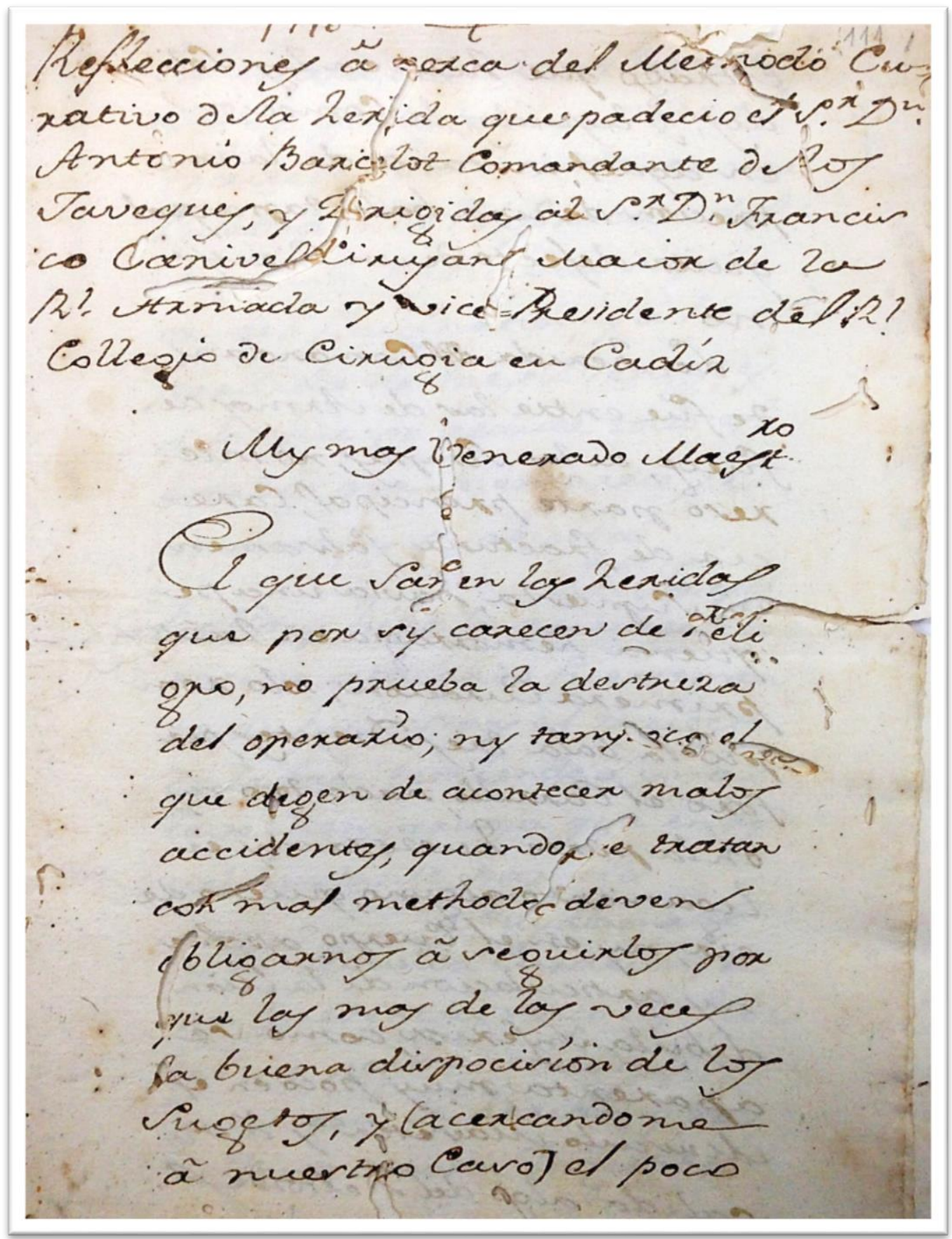

Fuente: Archivo de la Universidad de Cádiz (AUCA), C-36-99 RC

DOI del artículo:

https://doi.org/10.25267/Cuad investig fondos arch UCA.2021.i3.03 
Figura 4: Observación $n^{\circ}$ 131. «Observación de una Anasarca curada con el cocimiento de Retama y las Mostazas en la forma que se expresa». Por Juan de Navas; «Cadiz y Abril 28 de 1774»; censura de Domingo Castillejos del 27 de abril de 1775.

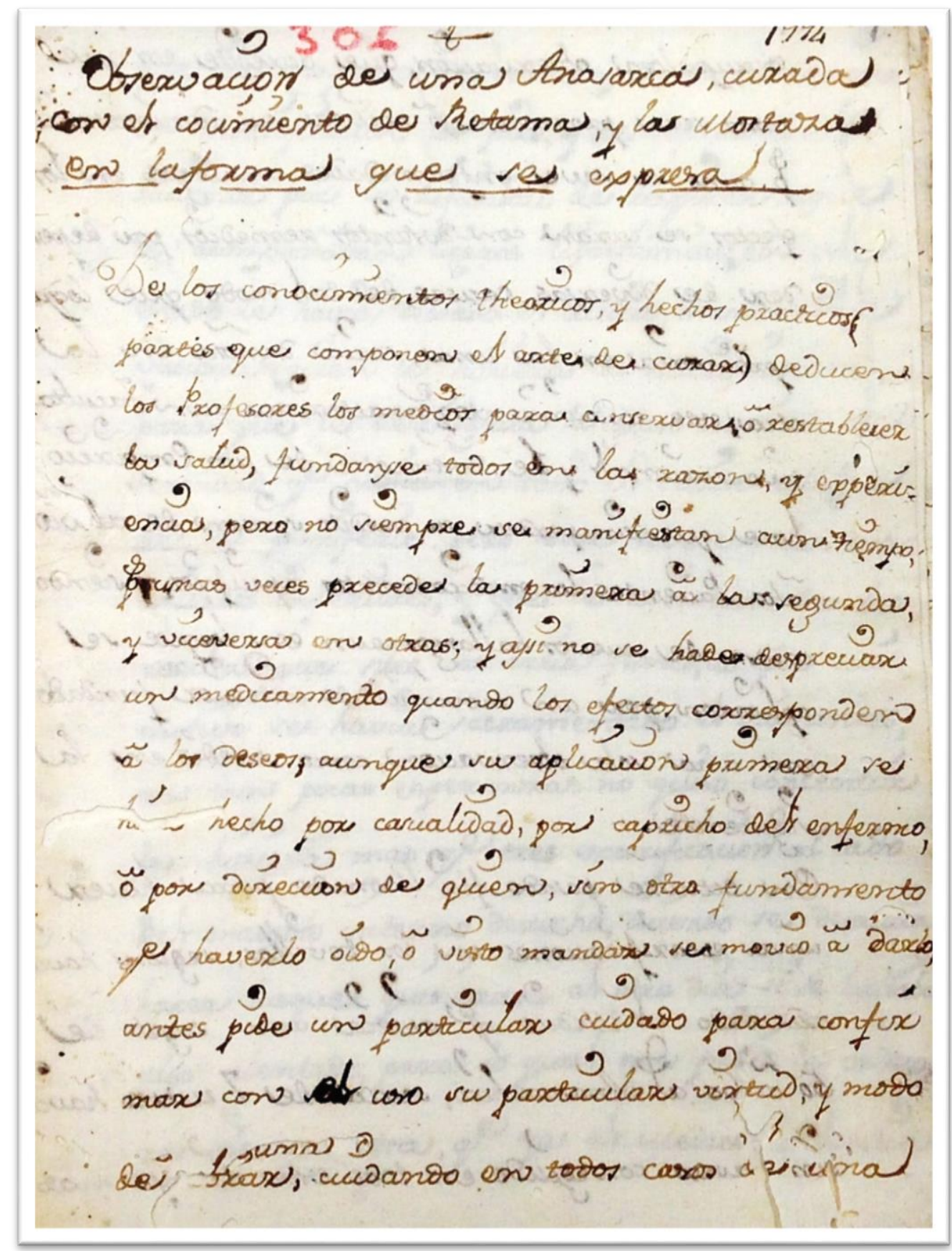

Fuente: Archivo de la Universidad de Cádiz (AUCA), C-36-131 RC

DOI del artículo: 
Figura 5: Observación n. ${ }^{\circ}$ 305. «Observación de una Amaurosis curada por la Medicina operatoria». Por José de Benjumeda del 7 de mayo de 1829. Censura de Ignacio Ameller del 28 de enero de 1830.

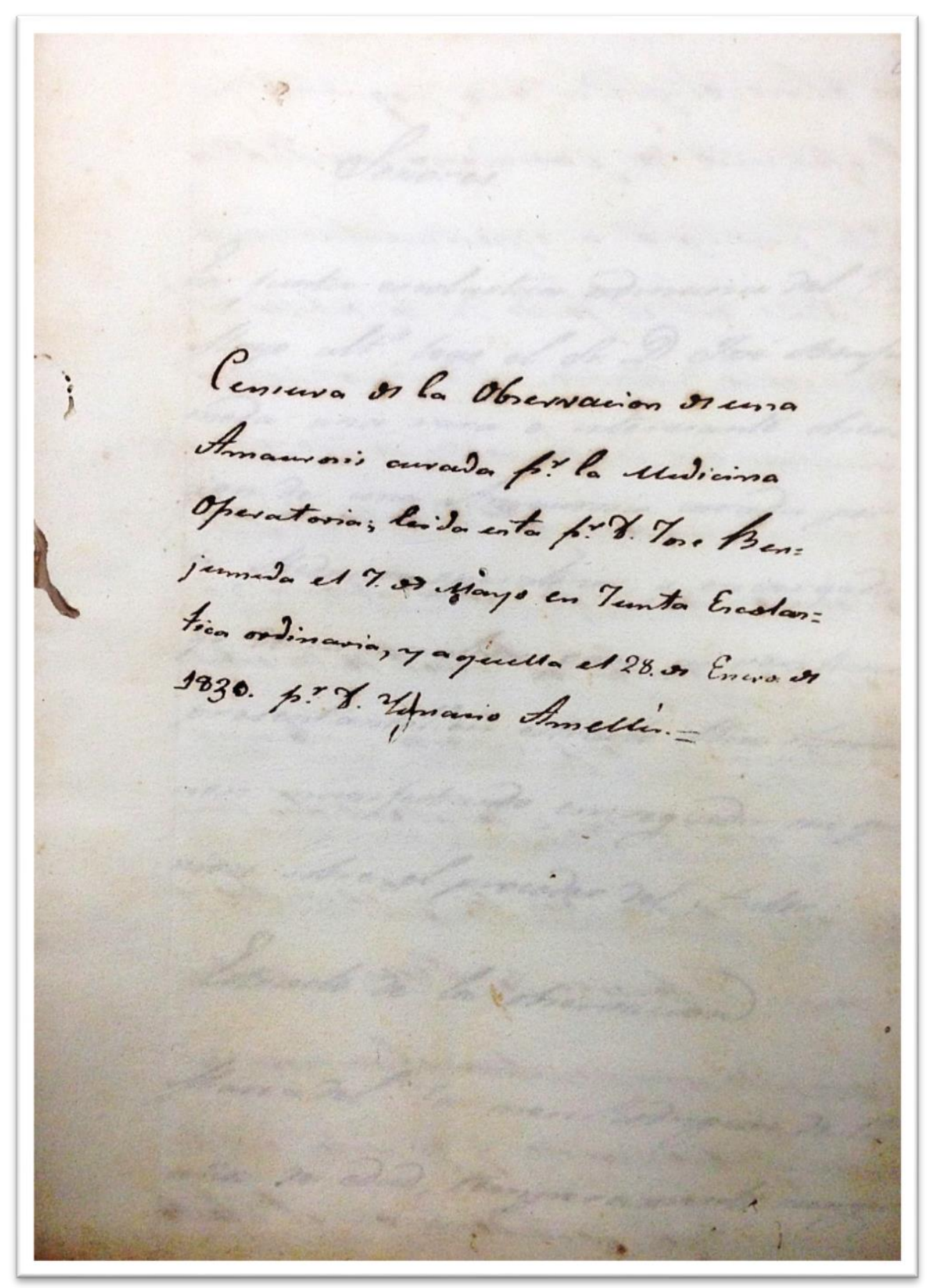

Fuente: Archivo de la Universidad de Cádiz (AUCA), C-39-305 RC 
Figura 6: Observación $n^{o}$ 30. «Methodo verosimil para proceder en la deliberacion de trepanar, en los casos de contusiones en el Craneo estando el hueso descubierto por bala û otro cuerpo empujado por arma de fuego contra la General y absoluta necesidad de trepanar en todas que establece Mr. Ledran». Por Francisco Canivell; "Cadiz y Diciembre a 18 de 1762».

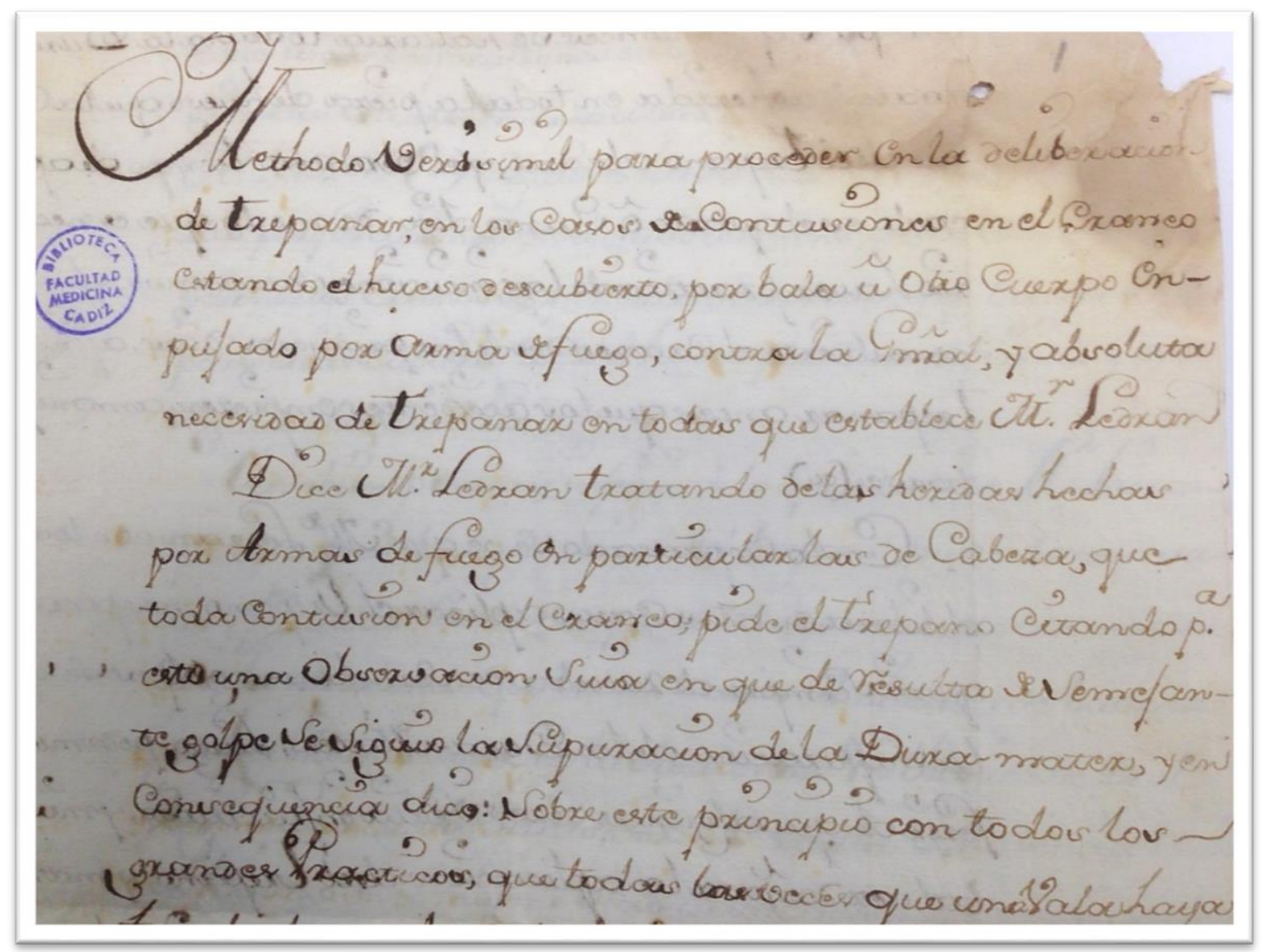

Fuente: Archivo de la Universidad de Cádiz (AUCA). C-35-30 RC 
Figura 7: Observación $n^{\circ}$ 57. «Obserbacion Anathomica sobre el no acostumbrado paso de la Arteria Cubital por delante de el Musculo Redondo Pronator». Por Vicente Lubet. Censura de Francisco Villaverde del 28 de abril de 1768.

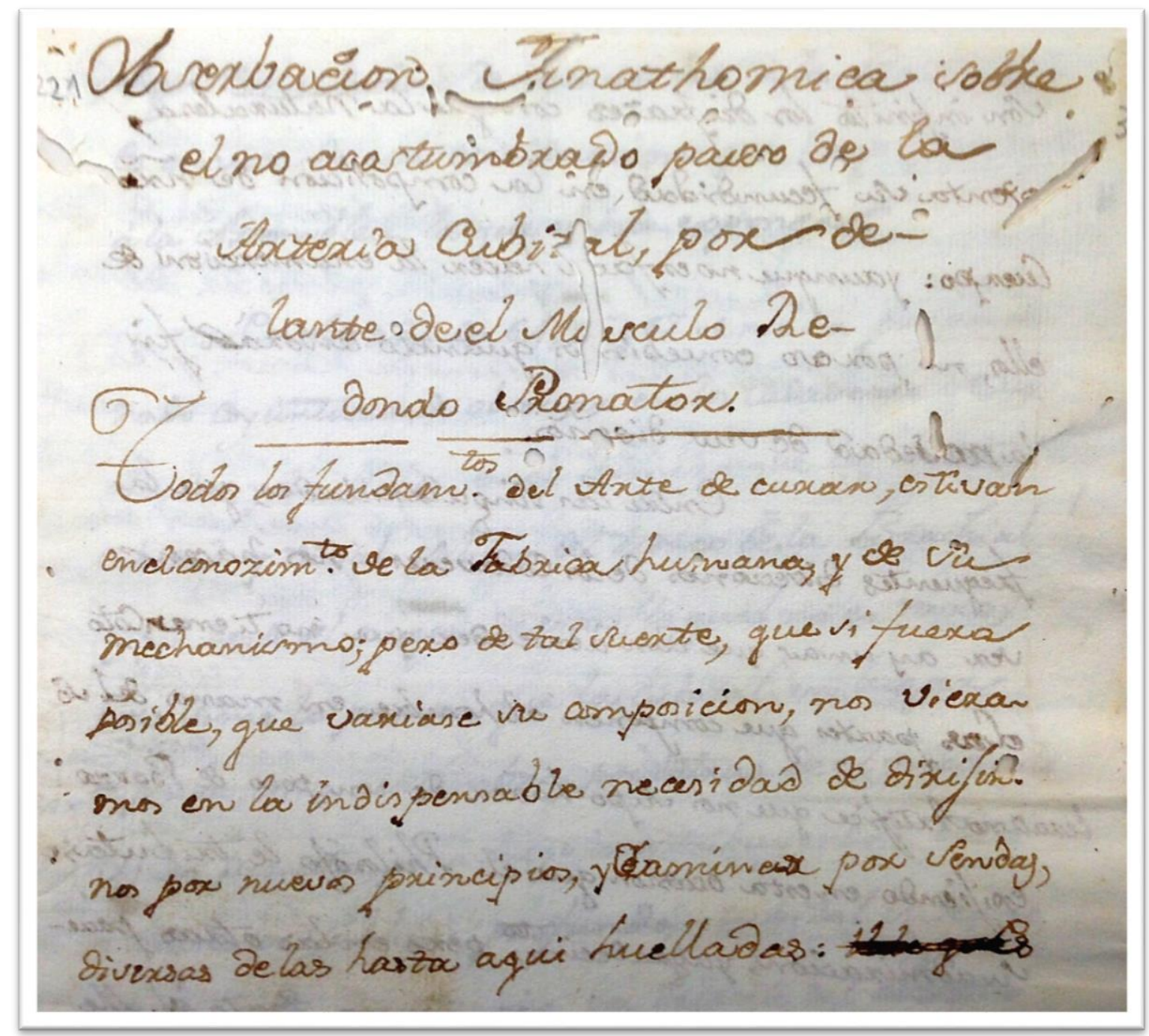

Fuente: Archivo de la Universidad de Cádiz (AUCA). C-35-57 RC

DOI del artículo: 
De esta forma, quedó constituida la estructura fundamental de las Observaciones clínicas del siglo XVIII y que con mayores o menores modificaciones fue practicada por los médicos y cirujanos de Europa, constituyendo una forma de enseñanza clínica recogida a la cabecera de los enfermos, con todos los detalles del tratamiento y publicadas posteriormente. Según el parecer de Renouard ${ }^{49}$, fueron de gran utilidad porque

... los hechos de la práctica diaria observados con atención y descritos con fidelidad, sirven para constituir la ciencia, para aumentarla a su vez, presentando en fórmulas abreviadas el resumen de la experiencia de todos los siglos, dirigiendo al práctico con una certidumbre cada vez mayor y ahorrándole infinitos tanteos y errores deplorables.

También, no cabe duda que esta forma de enseñanza clínica aprendida en Cádiz fue llevada por los alumnos del Real Colegio de Cirugía a sus diferentes destinos en Ultramar como ya hemos demostrado para el caso de Juan Miquel y Salazar ${ }^{50}$.

2. Observaciones constituidas por varias historias clínicas seguidas de reflexiones, correspondientes a casos clínicos enviados por los cirujanos navales desde sus puntos de destino; modalidad que no existe en las Juntas Literarias del Colegio de Barcelona ni en las Asambleas del Colegio de San Carlos de Madrid, y que constituyen un carácter exclusivo del Colegio de Cádiz.

3. Observación o Disertación doctrinal (fig. 6), sobre un punto concreto de doctrina, sin necesidad de historia clínica previa.

Por otra parte, la opinión de los cirujanos de la Armada sobre las Observaciones sólo está reflejada en tres de ellas; las numeradas $97^{51} ; 206^{52}$ y $236^{53}$ donde sus autores expresan que debían servir para el cumplimiento de las siguientes finalidades:

- A comunicar algunos fenómenos nuevos o pocas veces vistos del cuerpo humano de forma individual o asociada. Serían las denominadas observaciones raras (fig. 7).

\footnotetext{
${ }^{49}$ RENOUARD (1871): Historia de la medicina. Desde su origen hasta el siglo XIX, p. 608.

${ }^{50}$ MÁrquez Espinós, C., 2009: La formación médica de Juan Miquel y Salazar (1792-1866) en el Cádiz de las Cortes, pp. 86-91 y 171-184.

${ }^{51} C f$ : «Observacion comunicada, y leyda en la asamblea de 8 de $9^{\text {bre }}$ de 70 por $\mathrm{D}^{\mathrm{n}}$. Vicente Lubet». [Observación sobre un tumor en la rodilla]. Archivo de la Universidad de Cádiz (AUCA), C-36-97 RC.

52 «Disertacion Medico-Chirurgica, que sobre la amputacion de un brazo por la articulación con el Ômoplato, executada en el Real Ospital de Bellavista à las quatro y media de la tarde del dia veinte y uno de diciembre del año próximo pasado [1788], diò à luz, $\mathrm{D}^{\mathrm{n}}$. Miguel Josè Cabanellas. Ciruxano Mayor de dicho Ospital». Censura de Martín Ximénez y Pedro Belomo, fechada el 16 de octubre de 1788. AUCA, C-37-206 RC.

53 «Obcerbacion en $\mathrm{q}^{\mathrm{e}}$ se demuestra ser posible algunas veces averiguar las causas de las epidemias y de aquí el $\mathrm{q}^{\mathrm{e}}$ se puedan prevenir, y detener sus progresos» de Antonio García Puga, fechada en Cádiz el 17 de enero de 1793. Censura de Juan Manuel de Aréjula del 24 de enero de 1793. AUCA, C-38-236 RC.
}

DOI del artículo: https://doi.org/10.25267/Cuad investig fondos arch UCA.2021.i3.03 
- Aquellas observaciones que, en primer lugar, ilustran el curso de la enfermedad y detallan los esfuerzos de la naturaleza para superarla por sí misma o, en caso contrario, el resultado una vez instituido el tratamiento. Además, en este tipo de observación se debería reflexionar sobre la influencia que tuvo en el resultado final de la enfermedad el tratamiento aplicado y el momento oportuno para su aplicación.

- En el caso de las observaciones enviadas por los cirujanos embarcados o destinados en Ultramar servirían para constatar «el grado de adelantamiento que hayan adquirido desde su salida [del Real Colegio de Cirugía] ... y su aprobación o corrección para que rectifiquen su práctica».

- Finalmente, las observaciones debían enseñar a curar una o más enfermedades con el mismo tratamiento y esta repetición debía inducir en el médico la confianza suficiente en esta terapéutica para tratar las enfermedades semejantes.

\section{COROLARIO}

La publicación de las observaciones tal y como se detalla en el desarrollo normativo nunca fue llevada a cabo, sin embargo, a partir de su conocimiento por Diego Ferrer ${ }^{54}$, comenzó el interés por su estudio ${ }^{55}$, pero será el profesor Antonio Orozco Acuaviva, gaditano y catedrático de Historia de la Medicina de Cádiz, el que dará un importante impulso al conocimiento de estos documentos, no sólo con sus escritos ${ }^{56}$ sino, también, al promover bajo su magisterio la realización de Tesis de Licenciatura ${ }^{57}$ y Tesis de Doctorado ${ }^{58}$, asegurando la transcripción, el estudio y la edición de estos documentos fundamentales para el conocimiento de la medicina española de la Ilustración y el Romanticismo; labor que ha sido continuada por sus discípulos

\footnotetext{
${ }^{54}$ FERrer, D. (1961): Historia del Real Colegio de Cirugía de Cádiz, pp. 281-294.

55 Bustos Rodríguez, M (1983): Los Cirujanos del Real Colegio de Cádiz en la Encrucijada de la Ilustración (1748-1796).

${ }^{56}$ Entre otros, vid: OrozCo ACUAviva, A., 1975: «Una historia clínica anterior al Real Colegio de Cirugía de Cádiz». Ídem, 1978-1979: «Apuntes para la historia de la anatomía Gaditana del siglo XIX». Ídem, 1979: «Historia de una enfermedad de los navegantes: el Escorbuto. A propósito de dos «Observaciones» clínicas manuscritas de Joseph de Béjar (1776) y de Diego Terrero y Manuel de Padilla (1792)». Ídem, 1982: «Nuevos datos para el conocimiento de la Urología en la obra de Pedro Virgili (1699-1776). Ídem, 2000: Los cirujanos navales de la "Asamblea Amistosa Literaria” de Jorge Juan.

${ }^{57}$ Márquez Espinós, C., 1986: Las Juntas Literarias del Real Colegio de Cirugía de Cádiz. Catálogo de las «Observaciones» manuscritas (1742-1836).

${ }^{58}$ Cf:: MuÑOZ DE LA PASCUA, E., 1984: La patología de la generación en las observaciones manuscritas del Real Colegio de Cirugía de Cádiz. GARCíA CuRADO, L., 1984: La patología venérea en las observaciones manuscritas del Real Colegio de Cirugía de la Armada de Cádiz. LóPEZ DE COZAR, J. L., 1988: La aportación del Real Colegio de Cirugía de Cádiz al desarrollo de la urología española en la segunda mitad del siglo XVIII. Cózar Navarro, L., 1991: La patología infantil a través de las "observaciones" del Real Colegio de Cirugía de Cádiz.
}

DOI del artículo: https://doi.org/10.25267/Cuad investig fondos arch UCA.2021.i3.03 
ya sea por medio de publicaciones ${ }^{59}$ o por la realización de Tesis Doctorales ${ }^{60}$ bajo la dirección del Profesor Cabrera Afonso, sucesor del Profesor Orozco en la cátedra de Historia de la Medicina de la Facultad de Medicina de Cádiz.

Finalmente me queda expresar un deseo; creo que el estudio y la transcripción de estos documentos debe continuar para que no se pierda el esfuerzo ya realizado de conservación del patrimonio histórico-médico andaluz, en general, y gaditano, en particular, fundamental para el conocimiento de la medicina española de la Ilustración y el Romanticismo. Por estas razones, creo que sería deseable que el Servicio de Publicaciones de la Universidad de Cádiz (Editorial UCA) acogiera esta iniciativa de la edición crítica, ya comenzada por el grupo de discípulos del Prof. Orozco, de estos documentos que son patrimonio de todos.

Fuentes manuscritas del Real Colegio de Cirugía depositadas en el ARChivo HISTÓRICO DE LA UNIVERSIDAD DE CÁDIZ (AUCA)

Libro [Copiador] de Reales órdenes y decretos[...] en favor del Real Hospital Cirugía, Real Colegio Seminario de Cádiz e individuos que lo componen, L-54-RC.

Libro [cuarto] de Actas de la [Junta] del Colegio Nacional de Medicina y Cirugía, años: 18321836. L-25 RC.

Libro [tercero] de Actas de la [Junta] del Real Colegio de Cirugía y Real Colegio de Medicina y Cirugía, años 1825-1832. L-24-RC.

Libro de Noticias de propuestas y deliberaciones, así como de los gastos del Real Colegio de Cirugía, L-22-RC.

Observación $\mathrm{n}^{\circ}$ 206. «Disertacion Medico-Chirurgica, que sobre la amputacion de un brazo por la articulación con el Ômoplato, executada en el Real Ospital de Bellavista à las quatro y media de la tarde del dia veinte y uno de diciembre del año próximo pasado [1788], diò à luz, D ${ }^{n}$. Miguel Josè Cabanellas. Ciruxano Mayor de dicho Ospital». Censura de Martín Ximénez y Pedro Belomo, fechada el 16 de octubre de 1788. Archivo de la Universidad de Cádiz (AUCA), C-37-206 RC.

Observación $n^{\circ} 236$. «Obcerbacion en $q^{e}$ se demuestra ser posible algunas veces averiguar las causas de las epidemias y de aquí el $q^{e}$ se puedan prevenir, y detener sus progresos» de Antonio García Puga, fechada en Cádiz el 17 de enero de 1793. Censura de Juan Manuel de Aréjula del 24 de enero de 1793. Archivo de la Universidad de Cádiz (AUCA), C-38-236 RC.

\footnotetext{
${ }^{59}$ Cf.: CABRera Afonso, J. R., 2006: «El Real Colegio de Cirugía de Cádiz y el combate de Trafalgar». BlanCo Villero, J. M., 2007: Pedro María González Gutiérrez, médico-cirujano de la Real Armada. Biografía. MÁrquez ESPINós, C., 2009: La formación médica de Juan Miquel y Salazar (1792-1866) en el Cádiz de las Cortes.

${ }^{60}$ Vid.: Sepúlveda TosCano, A. F., 2004: La Materia Médica en el Real Colegio de Cirugía de la Armada a través de las "Observaciones" manuscritas (1790-1836). VACA HERNÁNDEZ, M., 2015: Vida y obra de Don Francisco de Flores Moreno. MÁRQUEZ-RodRíGUEZ, C. M., 2017: La reanimación del ahogado en la España ilustrada.
}

DOI del artículo:

https://doi.org/10.25267/Cuad investig fondos arch UCA.2021.i3.03 
Observación $n^{\circ}$ 97. «Observacion comunicada, y leyda en la asamblea de 8 de $9^{\text {bre }}$ de 70 por $D^{n}$.Vicente Lubet». [Observación sobre un tumor en la rodilla]. Archivo de la Universidad de Cádiz (AUCA), C-36-97 RC.

\section{FUENTES IMPRESAS}

ESTATUTOS, 1764: Estatutos y Ordenanzas Generales, que S.M. manda observar à los Colegios, y comunidades de Cirujanos, establecidos en Barcelona, Cádiz, y en todo el Principado de Cataluña, para la enseñanza de la Cirugía, Exámenes de los Proffesores, y su gobierno económico.

ORDENANZAS, 1791: Ordenanzas de S.M. que se deben observar en el Colegio de Medicina y Cirugía establecido en la ciudad de Cádiz, y por el cuerpo de sus profesores en la Real Armada, para gobierno del mismo Colegio, asistencia al Hospital, y servicio de los Buques de Guerra. Madrid, en la oficina de Don Benito Cano.

ORDENANZAS, 1795: Ordenanzas de S.M. que deben observarse por el Real Colegio de Cirugía de Barcelona, Cuerpo de Cirugía Militar, colegios subalternos y Cirujanos del principado de Cataluña. Madrid: en la Imprenta Real.

REAl CÉDUlA, 1828: Real Cédula de S.M. y Señores del Consejo, por la cual se manda observar en todo el Reino el nuevo Reglamento que ha tenido á bien aprobar para el régimen y gobierno de los colegios de Medicina y Cirugía, y de los Profesores que ejercen estas facultades. Madrid: en la Imprenta Real.

BIBLIOGRAFÍA

Aparicio Simón, J. (1956): Historia del Real Colegio de San Carlos de Madrid. Madrid: Aguilar.

ARAGÓN ESPESO, M. (2006): Cádiz, la medicina y la cirugía en la época de Trafalgar. Cádiz: Real Academia de Medicina y Cirugía de Cádiz.

Blanco Villero, J. M. (2007): Pedro María González Gutiérrez, médico-cirujano de la Real Armada. Biografía. Cádiz: Real Academia de Medicina y Cirugía de Cádiz.

Burke, M. E. (1977): The Royal College of San Carlos. Surgery and Spanish Medical Reform in the Late Eighteenth Century. Durham, N. C.: Duke University Press.

Bustos Rodríguez, M (1983): Los Cirujanos del Real Colegio de Cádiz en la Encrucijada de la Ilustración (1748-1796). Cádiz: Servicio de publicaciones de la Universidad de Cádiz.

Cabrera Afonso, J. R. (2006): «El Real Colegio de Cirugía de Cádiz y el combate de Trafalgar». En Trafalgar y Alcalá Galiano. Jornadas Internacionales. Cabra, 17 al 23 de octubre 2005. Madrid: Agencia Española de Cooperación Internacional, 253-275.

Clavijo y Clavijo, S. (1925): Historia del Cuerpo de Sanidad Militar de la Armada. San Fernando.

CózAr NAVARro, L. (1991): La patología infantil a través de las "observaciones" del Real Colegio de Cirugía de Cádiz. Tesis doctoral. Universidad de Cádiz. 
Ferrer, D. (1961): Historia del Real Colegio de Cirugía de Cádiz. Cádiz: Excmo. Colegio Oficial de Médicos.

--- (1966): «De la unión del estudio de la Medicina y la Cirugía». Medicina e Historia (24): 8-9.

--- (1983): Historia del Real Colegio de Cirugía de Cádiz. $2^{a}$ edic. facsímil. Cádiz: Servicio de Publicaciones de la Universidad de Cádiz.

GARCÍA CURADO, L. (1984): La patología venérea en las observaciones manuscritas del Real colegio de Cirugía de la Armada de Cádiz. Tesis doctoral. Universidad de Cádiz.

GeSTIDO DEL OlMO, R. (1994): Una Biblioteca ilustrada gaditana. Los fondos bibliográficos humanísticos del Real Colegio de Cirugía de la Armada. Cádiz: Servicio de Publicaciones de la Universidad de Cádiz.

LaÍn ENTRAlgO, P. (1961): La Historia clínica. Historia y teoría del relato patográfico. $2^{\mathrm{a}}$ edic. Barcelona: Editorial Salvat.

Lapuente Mateos, A. (1949): «Juntas Literarias y censuras en el Real Colegio de San Carlos». Archivos Iberoamericanos de Historia de la Medicina; 1: 404-418.

--- (1951): «Juntas Literarias y censuras en el Real Colegio de San Carlos». Archivos Iberoamericanos de Historia de la Medicina; 4: 575-581.

López de Cozar, J. L. (1988): La aportación del Real Colegio de Cirugía de Cádiz al desarrollo de la urología española en la segunda mitad del siglo XVIII. Tesis doctoral. Universidad de Cádiz.

Márquez Espinós, C. (1986): Las Juntas Literarias del Real Colegio de Cirugía de Cádiz. Catálogo de las «Observaciones» manuscritas (1742-1836). Cádiz: Servicio de Publicaciones de la Universidad de Cádiz.

--- (2009): La formación médica de Juan Miquel y Salazar (1792-1866) en el Cádiz de las Cortes. Cádiz: Real Academia de Medicina y Cirugía de Cádiz.

MÁRQUEZ-RodRíGUEZ, C. M. (2017): La reanimación del ahogado en la España ilustrada. Tesis doctoral. Universidad de Cádiz.

Massons, J. M. (2002): Història del Reial Col-legi de Cirurgia de Barcelona. Fundació Uriach 1838.

MuÑoz DE LA PASCUA, E. (1984): La patología de la generación en las observaciones manuscritas del Real Colegio de Cirugía de Cádiz. Tesis doctoral. Universidad de Cádiz.

Orozco Acuaviva, A. (1975): «Una historia clínica anterior al Real Colegio de Cirugía de Cádiz». Anales de la Real Academia de Medicina y Cirugía de Cádiz, 9(2): 109.

--- (1978-1979): «Apuntes para la historia de la Anatomía Gaditana del siglo XIX». Asclepio, 30-31: 99-117.

--- (1979): «Historia de una enfermedad de los navegantes: el Escorbuto. A propósito de dos «Observaciones» clínicas manuscritas de Joseph de Béjar (1776) y de Diego Terrero y Manuel de Padilla (1792)». Anales de la Real Academia de Medicina y Cirugía de Cádiz, 15(1): 8-27. 
--- (1982): «Nuevos datos para el conocimiento de la Urología en la obra de Pedro Virgili

(1699-1776)» Anales de la Real Academia de Medicina y Cirugía de Cádiz, 18(1): 2147.

--- (1985): «La biblioteca de la Facultad de Medicina de Cádiz entre Europa y América». En Actas del III Congreso nacional de Reales Academias de Medicina. Cádiz.

--- (1988): «El modelo de enseñanza en el Real Colegio de Cirugía de Cádiz en el siglo XVIII».

Gades; 18: 87-108.

--- (2000): Los cirujanos navales de la “Asamblea Amistosa Literaria” de Jorge Juan. Cádiz.

--- (2001): «Los Reales Colegios de Cirugía en España». En Rodríguez PÉREZ, E., MARTínEZ

BARbosa, X. (2001). Medicina Novohispana siglo XVIII. México: Universidad Nacional Autónoma de México.

Pérez PÉrez, A., MÁrquez Espinós, C. (2014): La medicina en la época de las Cortes de Cádiz. Cádiz: Publicaciones del Sur.

--- (2009): Juan Miquel y Salazar (1792-1866). Portorrealeño y pionero de la medicina chilena. Cádiz: Quorum editores.

PÉREZ-PÉREZ, N., SitGeS-SERRA, A. (2010): «Juntas Literarias: legado de la cirugía ilustrada». Cirugía Española; 87(1): 9-12.

Remón Rodríguez, A. (2017): El libro médico-científico en la Biblioteca del Real Colegio de Cirugía de Cádiz. Cádiz: Editorial UCA.

Renouard, P. V. (1871): Historia de la medicina. Desde su origen hasta el siglo XIX. Traducida, adicionada y anotada por D. Pablo Villanueva. Salamanca: Imprenta de Sebastián Cerezo.

Usandizaga, M. (1948): Historia del Real Colegio de Cirugía de San Carlos de Madrid (1787-1828). Madrid: Consejo Superior de Investigaciones Científicas.

--- (1956): Juntas Literarias del Real Colegio de Cirugía de Barcelona. Barcelona.

Usandizaga Soraluce, M. (1964): Historia del Real Colegio de Cirugía de Barcelona (1760-1843). Barcelona: Instituto Municipal de Historia.

VACA Hernández, M. (2015): Vida y obra de Don Francisco de Flores Moreno. Tesis Doctoral. Universidad de Cádiz. 